\title{
Circular RNA YAP1 inhibits the proliferation and invasion of gastric cancer cells by regulating the miR-367-5p/p27 Kip1 axis
}

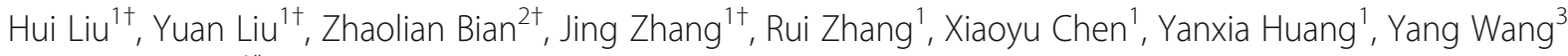
and Jinshui Zhu ${ }^{1 *}$

\begin{abstract}
Background: Circular RNAs (circRNAs) are a new type of non-coding RNAs and their functions in gastric cancer (GC) remain unclear. Recent studies have revealed that circRNAs play an important role in cancer development and certain types of pathological responses, acting as microRNA (miRNA) sponges to regulate gene expression.

Methods: CircNet was used to screen potential circRNAs and validated circYAP1 expression levels in 17 GC tissues by quantitative real-time PCR (qRT-PCR) and another 80 paired GC tissues by FISH. CircYAP1 overexpression and knockdown experiments were conducted to assess the effects of circYAP1 in vitro and in vivo, and its molecular mechanism was demonstrated by RNA in vivo precipitation assays, western blotting, luciferase assay and rescue experiments.

Results: CircYAP1 expression level was significantly lower in GC tissues than the adjacent normal tissues, and GC patients with circYAP1 low expression had shorter survival times as compared with those with circYAP1 high expression. Functionally, circYAP1 overexpression inhibited cell growth and invasion in vitro and in vivo, but its knockdown reversed these effects. Further analysis showed that circYAP1 sponged miR-367-5p to inhibit p27 Kip1 expression and GC progression.

Conclusion: Our findings demonstrate that circYAP1 functions as a tumor suppressor in GC cells by targeting the miR-367-5p/p27 Kip1 axis and may provide a prognostic indicator of survival in GC patients.
\end{abstract}

Keywords: circYAP1, Gastric cancer, Growth, Invasion, miR-367-5p

\section{Background}

Gastric cancer (GC) continues to be a major threat to human health and it is the fourth most common cancer and the third-leading cause of cancer-related deaths worldwide according to global cancer statistics [1]. Despite the application of many advances in diagnosis and treatment, the prognosis of $\mathrm{GC}$ remains relatively poor, with a 5 -year overall survival below $40 \%$ in most countries, due to tumor metastasis and recurrence [2]. In the past decades, non-coding RNAs (ncRNAs), including microRNA (miRNA) and long non-coding RNA

\footnotetext{
*Correspondence: zhujs1803@163.com

${ }^{+}$Hui Liu, Yuan Liu, Zhaolian Bian and Jing Zhang contributed equally to this work.

'Department of Gastroenterology, Shanghai Jiao Tong University Affiliated Sixth People's Hospital, No. 600 Yishan Road, Shanghai 200233, China Full list of author information is available at the end of the article
}

(lncRNA) have been deregulated in GC patients, and have potential clinical applications [3, 4]. Recent studies have shown that circular RNAs (circRNAs) are aberrantly expressed in GC, lung cancer, hepatocellular carcinoma (HCC) and colorectal cancer (CRC), involved in cancer development [5]. Therefore, it is essential to identify deregulated circRNAs and discover novel molecular mechanisms and therapeutic targets for the treatment of GC.

CircRNAs are a special type of ncRNAs derived from exons, introns or intergenic regions that are covalently linked to form a closed circular structure without $5^{\prime}$ caps and $3^{\prime}$ tails, display cell or tissue-specific expression, and are conserved across species due to their resistance to RNase R [6-8]. Compared with linear RNAs, circRNAs are remarkably stable, and accumulate 
primarily in the cytoplasm, acting crucial roles in human diseases $[9,10]$. Emerging evidence shows that circRNAs act as miRNA sponges to regulate gene expression and interact with RNA binding proteins (RBPs) [8, 11]. However, the functions of the newly identified circRNAs in special fields require further investigation.

CircRNAs participate in a wide range of biological processes, including transcription, mRNA splicing, RNA decay and translation, and their dysregulation leads to abnormal cellular functions and human diseases [12]. It is revealed that certain types of circRNA are deregulated in HCC, CRC, esophageal squamous cancer, oral cancer and bladder cancer, and are associated with cancer progression [13-17]. Those studies indicate that circRNAs may be potential biomarker and therapeutic target for cancer.

In our study, we selected a circRNA, termed circYAP1 (has_circ_0002320) by CircNet (http://syslab5.nchu.edu.tw/CircNet/) and validated that circYAP1 expression level was dramatically decreased in GC tissues. Low expression of circYAP1 was associated with the poor prognosis of patients with GC. More importantly, we found that circYAP1 functioned as a sponge of oncogenic miR-367-5p to upregulate p27 Kip1 and consequently suppressed the tumorigenesis of GC.

\section{Methods}

\section{Clinical data and tissues}

A human tissue microarray of 80 paired GC patients (Cat No. STC1602) was purchased from Shanghai Superbiotek Pharmaceutical Technology Co. Ltd. (Shanghai, China). Seventeen GC and paired adjacent gastric tissue samples were obtained from patients undergoing surgery at Nantong Third People's Hospital Affiliated to Nantong University. All samples were collected with consent from the patients and were stored at $-80{ }^{\circ} \mathrm{C}$ until use. All experiments were approved by the Ethics Committee of Shanghai Jiao Tong University Affiliated Sixth People's Hospital and Nantong Third People's Hospital Affiliated to Nantong University.

\section{Identification of miRNAs sponged by circYAP1}

The miRNAs that might be sponged by circYAP1 were identified by using CircNet and Circular RNA Interactome, of which the top 3 miRNAs (miR-367-5p, miR-513c-3p and miR-513a-3p) sponged by circYAP1 were selected through the CircNet, and another $3 \mathrm{miR}$ NAs (miR-1200, miR-330-5p, and miR-513a-3p) were chosen by the Circular RNA Interactome. Therefore, miR-513a-3p could be predicted to sponge circYAP1 by both CircNet and Circular RNA Interactome, and these miRNAs were further confirmed by qPCR in circYAP1 overexpressed cells.
RNA fluorescence in situ hybridization (FISH)

An oligonucleotide-modified probe sequence for human circYAP1 (3'-CCTCTTCCTCTCCGACGCCGACTTTG TCGTTCTTGACGAAGCCGTCCAGGAGAAGGACTA CCTACCCTTGTTCGGTACTGAGTCCTACCTCTTT

A-5') was used for FISH. The probe of circYAP1 was marked with DIG-UTP (Roche,11,209,256,910) for RNA labeling. After dehydration with 70, 95 and 100\% ethanol, hybridization was carried out at $37{ }^{\circ} \mathrm{C}$ overnight in a dark moist chamber. After hybridization, slides were washed three times in 50\% $60 \mathrm{ml}$ formamide/2X SSC for $5 \mathrm{~min}$ and was incubated with anti-DIG-HRP (PerkinElmer, NEF832001EA) at $4{ }^{\circ} \mathrm{C}$ overnight, after being washed for 3 times for $10 \mathrm{~min}$ at room temperature, the slides were incubated with TSA fluorescent signal reaction solution (PerkinElmer, NEL701001KT, TSA Fluorescein system) for $30 \mathrm{~min}$ and was sealed with tablets containing DAPI. The images were acquired using a fluorescence microscopy (Leica, SP8 laser confocal microscopy). The analysis software Image-pro plus 6.0 (Media Cybernetics, Inc., Rockville, MD, USA) was applied to acquire the Immunofluorescence Accumulation Optical Density (IOD) for evaluating the expression level of circYAP1 in GC tissues.

\section{CircYAP1 and miR-367-5p co-location by RNA fluorescence in situ hybridization (FISH)}

The probe for circYAP1 was 5'-FAM-ATCAG GAAGA GGACC TGCCG AAGCA GTTCT-FAM-3', and the probe for miR-367-5p was 5'-CY3-AGAGT TGCAT ATTAG CAACA GT-CY3-3'. After dehydration with 70, 95 and 100\% ethanol, hybridization was carried out at $37{ }^{\circ} \mathrm{C}$ overnight in a dark moist chamber. After each hybridization, slides were washed three times in $50 \% 60 \mathrm{ml}$ formamide/2X SSC for 5 min respectively. The images were acquired using a fluorescence microscopy (Leica, SP8 laser confocal microscopy). The analysis software Image-pro plus 6.0 (Media Cybernetics, Inc., Rockville, MD, USA) was applied to acquire the Immunofluorescence Accumulation Optical Density (IOD) for evaluating the expression level of circYAP1 and miR-367-5p in GC tissues.

\section{Cell culture}

The normal human gastric epithelial cell line GES-1 and a GC cell line (HGC-27) were purchased from the Cell Laboratory of Central South University. MKN-45 and AGS cell lines were obtained from the Digestive Disease Laboratory of Shanghai. GES-1 and GC cell lines were cultured in Dulbecco's modified Eagle's medium (DMEM) (Gibco, Rockford, MD, USA) or 1640 medium (Gibco) supplemented with $10 \%$ fetal bovine serum (FBS), $100 \mathrm{U} / \mathrm{ml}$ penicillin, and $100 \mu \mathrm{g} / \mathrm{ml}$ streptomycin. All cells were cultured in a humidified atmosphere containing $5 \% \mathrm{CO}_{2}$ at $37{ }^{\circ} \mathrm{C}$. 


\section{Quantitative real-time PCR (qRT-PCR)}

Total RNA was extracted using RNAiso Plus (TaKaRa, Dalian, China), reverse transcription was performed using PrimeScrip ${ }^{\text {tw }}$ RT Master Mix (TaKaRa), and cDNA amplification was performed using SYBR Green Premix Ex Taq ${ }^{\mathrm{Tm}}$ II (Takara) according to the manufacturer's instructions. miRNA was extracted using an miRNeasy Mini Kit (Qiagen, Duesseldorf, Germany), reverse transcription was performed using an miScript II RT Kit (Qiagen), and cDNA amplification was performed using an miScript SYBR Green PCR Kit (Qiagen) according to the manufacturer's instructions. The primers are listed in Additional file 1: Table S1.

\section{CircRNA in vivo precipitation (circRIP)}

A biotin-labeled circYAP1 probe was designed and synthesized by GenePharma. The sequence was as follows: 5'-GAA CTG CTT CGG CAG GTC CTC TTC CTG A-3'-biotin; the circRIP assay was performed according to the reported literature with minor alterations $[13,18]$. CircYAP1-overexpressing AGS cells were seeded in a $10-\mathrm{cm}$ dish at a density that allowed them to grow for $48 \mathrm{~h}$ without reaching complete confluency. Then, the cells were transfected with the specific biotin-tagged probe or control probe at a final concentration of $200 \mathrm{nmol} / \mathrm{L}$. The cells were harvested after transfection for $24 \mathrm{~h}$. Then, the cells were cross-linked with $1 \%$ formaldehyde for $10 \mathrm{~min}$, lysed and sonicated. After centrifugation, $50 \mu \mathrm{L}$ of the supernatant was retained as input, and the remaining cell lysis solution was incubated with a circYAP1-specific probe-streptavidin Dynabeads (M-280, Invitrogen, CA, USA) mixture overnight at room temperature. The next day, the M-280 Dynabead-probe-circRNA mixture was washed and incubated with $200 \mu \mathrm{L}$ of lysis buffer and proteinase $\mathrm{K}$ to reverse the formaldehyde crosslinking. Finally, the mixture was extracted to obtain the total RNA using the miRNeasy Mini Kit according to the manufacturer's instructions (Qiagen).

\section{Lentiviral vector, siRNA, mimics and inhibitors}

The lentivirus-mediated circYAP1 overexpression vector and no-load lentivirus vector were supplied by Hanbio (Shanghai, China). GC cells (MKN-45 and AGS) were infected with the lentivirus according to the manufacturer's instructions, and stable cells were selected with puromycin. miR-367-5p mimics and inhibitors were synthesized by GenePharma (GenePharma, Suzhou, China). The siRNA sequence that targeted circYAP1 for knockdown was as follows: CGG CAG GUC CUC UUC CUG ATT; this sequence was designed and synthesized by GenePharma. The mimics, inhibitor and siRNA were transfected with Lipofectamine 2000 (Invitrogen) according to the manufacturer's instructions.

\section{Cell proliferation assay}

Cell proliferation was detected using a CCK- 8 assay kit (Dojindo Corp, Japan). A total of 3000 cells were plated in each well of a 96-well plate. Then, on the indicated day, $10 \mu \mathrm{L}$ of CCK- 8 reagent was added directly to the culture medium. Then, the cells were incubated for $2.5 \mathrm{~h}$ at $37^{\circ} \mathrm{C}$, and the optional density was measured at $450 \mathrm{~nm}$. These experiments were repeated three times.

\section{5-Ethynyl-20-deoxyuridine (EdU) incorporation assay}

EdU assays were performed using a Cell-Light EdU DNA Cell Proliferation Kit (RiboBio, Guangzhou, China) according to the manufacturer's instructions. A total of $1 \times 10^{4}$ cells were seeded in each well of a 96-well plate. After incubation with $50 \mu \mathrm{M}$ EdU for $2 \mathrm{~h}$, the cells were fixed in $4 \%$ paraformaldehyde and stained with Apollo Dye Solution. Hoechst-33,342 was used to stain the nucleic acids. Images were acquired using an Olympus IX73 microscope (Olympus, Tokyo, Japan), and the percentage of EdU-positive cells was calculated using ImageJ (National Institutes of Health, Bethesda, MD, USA). These experiments were repeated three times.

\section{Colony formation assay}

After MKN-45 and AGS cells were transfected with the indicated lentivirus, mimics, inhibitor or siRNA for $48 \mathrm{~h}$, a total of $1 \times 10^{3}$ cells were seeded in a 6-well plate and incubated at $37{ }^{\circ} \mathrm{C}$ for 7 days, and the medium was changed every two days. On day 7 , all the cells were fixed in $4 \%$ paraformaldehyde and dyed with a crystal violet solution. Cell colonies were then counted and analyzed. These experiments were repeated three times.

\section{Transwell invasion assay}

The cell invasion assay was carried out using 24-well Transwell (Corning Costar, $8.0 \mu \mathrm{m}$ pore size) insert chamber plates. The upper surfaces of Transwell filters were coated with Matrigel (BD, New Jersey, USA). Transfected cells $\left(3 \times 10^{5}\right)$ in $200 \mu \mathrm{l}$ of serum-free 1640 medium were added to the upper compartment of the chamber. A total of $500 \mu \mathrm{L}$ of 1640 medium supplemented with $10 \%$ FBS was added into the lower chamber. The invaded cells were harvested after incubation for $48 \mathrm{~h}$. The non-invaded cells on the upper side of the chamber were scraped off with a cotton swab. The cells were fixed in $4 \%$ paraformaldehyde and stained with a crystal violet solution. The cells were then counted and analyzed. These experiments were repeated three times.

\section{Western blotting analysis}

MKN-45, AGS and HGC-27 cells were harvested and extracted using RIPA lysis buffer (Beyotime, Shanghai, China), and equal amounts of cells extracts were separated on $12 \%$ SDS-PAGE gels. The separated protein 
bands were transferred onto nitrocellulose (NC) membranes. The primary antibodies anti-p27 ${ }^{\text {Kip1 }}$ (Cell Signaling Technology, MA, USA) and anti- $\beta$-actin (Proteintech, IL, USA) were diluted 1:1000 according to the instructions and incubated overnight at $4{ }^{\circ} \mathrm{C}$. HRP rabbit IgG secondary antibodies were added at a dilution of 1:2000 and incubated for $1 \mathrm{~h}$ at room temperature. The membranes were washed three times with TBST, and the immunoreactive bands were visualized using SuperSignal West Dura Extended Duration Substrate (Thermo Fisher, IL, USA) according to the kit's instructions. These experiments were repeated three times.

\section{Luciferase reporter assay}

293T cells were seeded into 96-well plates. The fragments including the 3'-UTR wild-type (P27-WT) regions of $\mathrm{p} 27^{\mathrm{Kip} 1}$ were cloned into SacI/Sall-digested pmirGLO vector (Promega, USA), which included both Renilla and Firefly luciferase reporter genes. The mutant p27 ${ }^{\text {Kip } 1} 33^{\prime}$-UTR (P27-MUT) was generated by mutating the conserved binding sites for miR-367-5p using Gene Mutation Kit (Takara, JAPAN). Then 60 ng P27-WT or P27-MUT plasmid were co-transfected with miR-367-5p mimics into $293 \mathrm{~T}$ cells. The miR-NC mimics was used as negative control. After $48 \mathrm{~h}$, the cells were harvested, and the Firefly and Renilla luciferase activities were measured with a dual-luciferase reporter assay system (Promega, USA). The relative luciferase activity was normalized to Renilla luciferase activity.

\section{Flow cytometric analysis}

To detect cell apoptosis, GC cells were digested, washed with cold PBS and resuspended in binding buffer according to the instructions of the apoptosis kit. APC-AnnexinV and 7-AAD were added to the fixed cells for $15 \mathrm{~min}$ in the dark at room temperature. Then, Annexin $\mathrm{V}$ binding buffer was added to the mixture before the fluorescence was measured with a BD FACSCalibur flow cytometer (BD). Cell apoptosis was analyzed using Cell Quest software (Becton Dickinson, USA). Three separate experiments were performed.

To detect cell cycle, cells were seeded in a six-well plate at $5 \times 10^{5}$ cells per well. After transfection, the cells were maintained for $48 \mathrm{~h}$ before harvest. Harvested cells were washed with PBS and fixed with $70 \%$ ethanol. The fixed cells were stained with PI in the presence of RNase A for $15 \mathrm{~min}$ at room temperature in the dark. The samples were then analyzed with a BD FACSCalibur flow cytometer (BD) after acquiring 10000 events for each sample. FlowJo V10 software was used for the cell cycle analyses. Three separate experiments were performed.

\section{Tumor model in mice}

NOD/SCID mice were purchased from the Shanghai Laboratory Animal Central (SLAC, Shanghai, China). Approval for the experiments was obtained from the Institutional Animal Care and Use Committee of Shanghai Jiao Tong University Affiliated to Shanghai Sixth People's Hospital. MKN-45 cells $\left(1 \times 10^{7}\right)$ transfected with the circYAP1 overexpression vector or no-load lentivirus vector were resuspended in PBS and injected subcutaneously into the right front armpits of 6-week-old mice. After 4 weeks, the mice were sacrificed, and the xenografted tumors were collected to calculate the volume and for further experiments.

\section{Statistical analysis}

Statistical analyses were carried out using SPSS 20.0(IBM, SPSS, Chicago, IL, USA) and GraphPad Prism (GraphPad, La Jolla, USA). Student's t-test or Chi-square test was used to assess the statistical significance for comparisons of two groups. OS was defined as the interval between the dates of surgery and death, and OS curves were analyzed with the Kaplan-Meier method and log-rank test. Univariate analysis and multivariate models were performed using a Cox proportional hazards regression model. Receiver operating characteristic (ROC) curves were obtained using cutoff Finder online software (http://molpath.charite.de/cutoff/load.jsp). $P<0.05$ was considered statistically significant.

\section{Results}

\section{Low expression of circYAP1 indicates poor prognosis in GC patients}

We measured the expression of circYAP1 in 17 patients with GC by qPCR. Compared with that in adjacent normal tissues, circYAP1 expression was significantly decreased from 1 to 0.09 in $76.5 \%$ (13 of 17 ) of GC tissues (Fig. 1a, $P=0.0383$ ). We further confirmed this result by FISH assay using tissue microarray in $80 \mathrm{GC}$ samples from patients with survival data (Additional file 1: Table S2). As shown in Fig. 1b, circYAP1 was dramatically lower in GC tissues than in adjacent normal tissues. In addition, we found that circYAP1 expression was lower in GC patients with a tumor size (TS) $\geq 5 \mathrm{~cm}$ than those with a TS $<5 \mathrm{~cm}$ (Additional file 2: Figure S1a). Compared with that in GC tissue, the circYAP1 expression level in adjacent normal tissue was higher in $59 \mathrm{GC}$ patients and lower in $21 \mathrm{GC}$ patients (Fig. $1 \mathrm{c}, P<0.0001$ ). To illustrate the relationship between circYAP1 and GC, we divided the GC patients into four groups according to the TNM staging system. CircYAP1 expression was dynamically altered with the GC stage, and it was higher in patients with early-stage GC than those with advanced stage GC (Fig. 1d). 
a

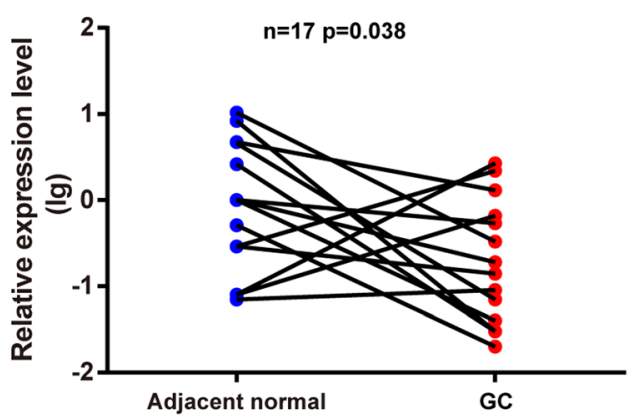

C

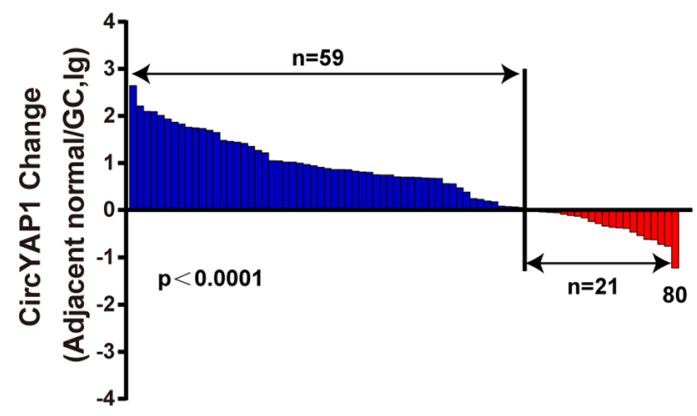

e

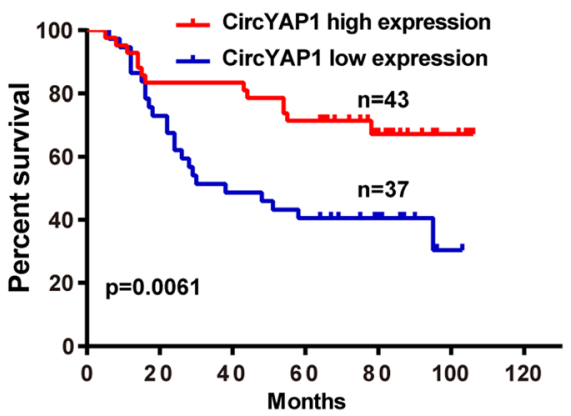

g

With Chemotherapy

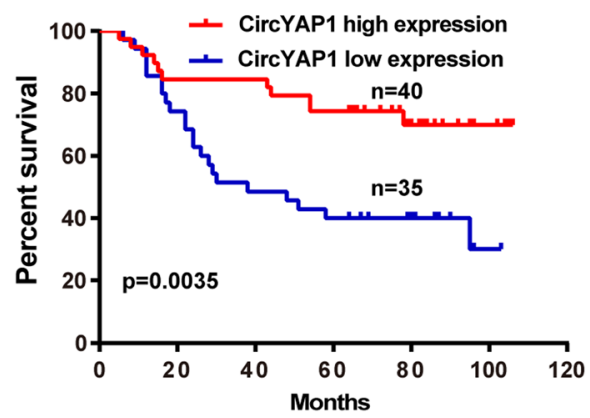

b

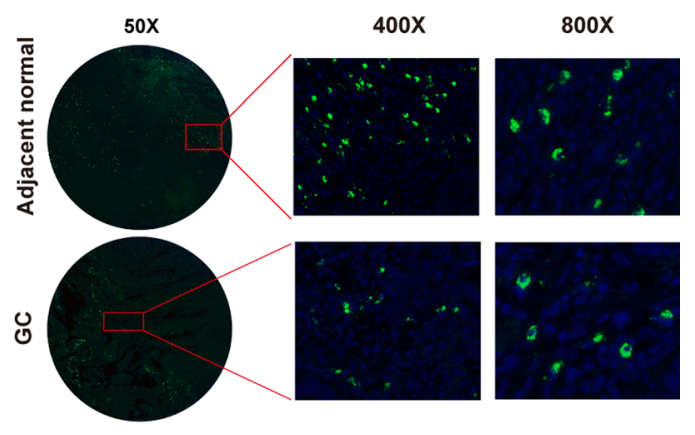

d

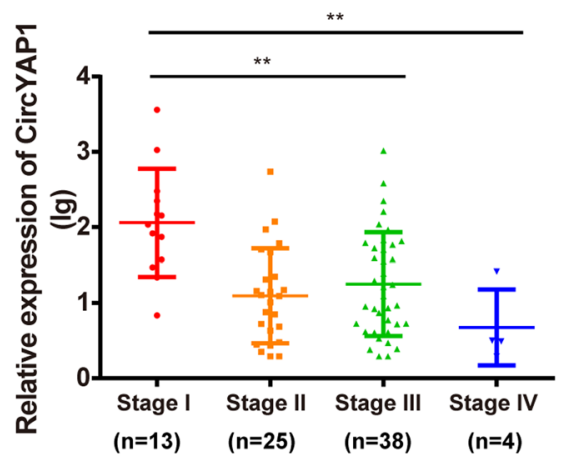

f

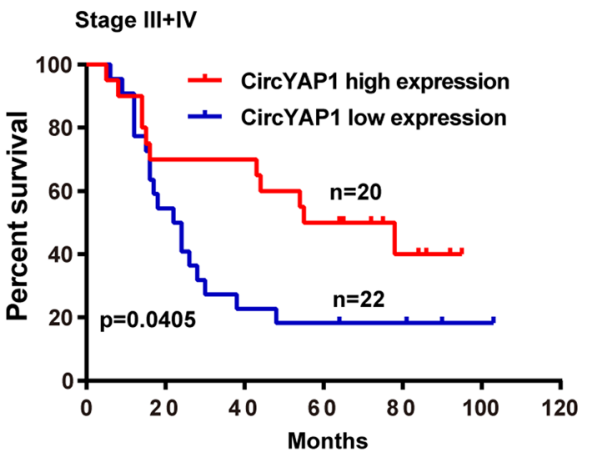

h

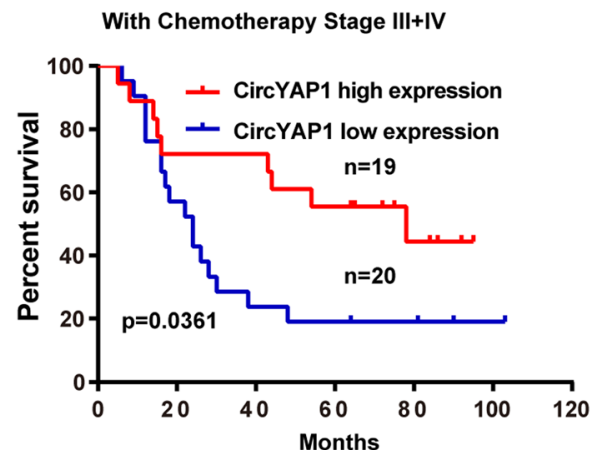

Fig. 1 (See legend on next page.) 
(See figure on previous page.)

Fig. 1 CircYAP1 acts as an independent prognostic factor for OS in GC patients. a, CircYAP1 expression was significantly lower in GC tissues than in normal adjacent tissues from 17 patients with GC according to GPCR. b. Cellular localization of circYAP1 in GC tissue cells and adjacent normal tissue cells. The nuclei was stained blue with DAPI, and cytoplasmic circYAP1 was stained green. c, CircYAP1 expression levels were lower in GC tissues than in normal tissues. $\mathbf{d}$, CircYAP1 expression levels were higher in early-stage GC patients than in advanced stage GC patients. e-f, Kaplan-Meier analysis of the association of circYAP1 expression levels with the OS of GC patients and late-stage GC patients (stage III + IV). $\mathbf{g}$-h Kaplan-Meier analysis of the association of circYAP1 expression with the therapeutic outcomes of GC patients and late-stage GC patients (stage III + IV). ${ }^{*} P<0.05$; ${ }^{* *} P<0.01$

Next, we analyzed whether circYAP1 can act as an independent prognostic factor for GC patients. The GC patients were divided into two groups, circYAP1 high expression and circYAP1 low expression, according to the cutoff value, which was defined by Cutoff Finder (Additional file 2: Figure S1b). High circYAP1 expression was negatively associated with tumor size but was not correlated with other pathological parameters (Additional file 1: Table S3).

Survival analyses were performed using log-rank tests. GC patients with high circYAP1 expression had longer overall survival (OS) times than those with low circYAP1 expression (Fig. 1e, $P=0.0061$ ). In addition, advanced stage (stage III + IV) patients with high circYAP1 expression had longer OS times than those with low circYAP1 expression (Fig. 1f, $P=0.0405$ ). Furthermore, we analyzed the association between circYAP1 expression levels and therapeutic outcomes in $75 \mathrm{GC}$ patients treated with adjuvant chemotherapy (oxaliplatin and 5-Fu). We found that the GC patients with high circYAP1 expression had more favorable therapeutic outcomes than those with low circYAP1 expression (Fig. 1g, $P=0.0035$ ). The same result was observed for the advanced stage patients (Fig. $1 \mathrm{~h}, P=0.0361$ ). To determine whether circYAP1 is an independent prognostic factor of OS in GC patients, univariate and multivariate Cox proportional hazards analyses were performed. CircYAP1 level, tumor size and lymphatic metastasis were independent prognostic factors for the OS of GC patients (Additional file 1: Table S4).

\section{CircYAP1 was confirmed to sponge MiR-367-5p in GC cells}

As previously reported, circRNAs primarily function as miRNA sponges to regulate gene expression. Therefore, we examined the potential miRNAs associated with circYAP1. CircInteractome and CircNet were used to predict the potential target miRNAs that could bind with the circYAP1 sequence, and five miRNAs were selected as the best potential targets of circYAP1. To investigate whether these five miRNAs can interact with circYAP1 in GC cells, we first overexpressed circYAP1 in the AGS and MKN-45 cell lines and performed qPCR to measure the following miRNAs: miR-1200, miR-330-5p, miR -367-5p, miR-513a-3p and miR-513c-3p. The results showed that miR-1200 and miR-367-5p were significantly upregulated by circYAP1 in the AGS cells $(P=$ $0.0161 ; P=0.0238$ ), and the expression levels of the other miRNAs were not changed (Fig. 2a). In addition, only miR-367-5p expression was increased by circYAP1 in the MKN-45 AGS cells (Fig. $2 b, P=0.0113$ ). Therefore, miR-367-5p might be bound to circYAP1 in GC cells. To confirm this hypothesis, we used a circYAP1-specific probe to perform RNA in vivo precipitation (RIP) which has been reported in several studies [13, 18]. We purified circYAP1-associated RNA using a circYAP1 specific-probe and performed qPCR to measure the expression of circYAP1 and miR-367-5p. qPCR showed the enrichment of circYAP1 and miR-367-5p compared to the controls (Fig. 2c and d, $P=0.0050 ; P=$ 0.0224 ), indicating that miR-367-5p was sponged by circYAP1 in GC cells. The binding sites of miR-367-5p to circYAP1 were shown in Fig. 2e. By using FISH analysis in GC tissues, we found that circYAP1 and miR-367-5p were co-localized in the cytoplasm (Fig. 2f). The expression of circYAP1 had the positive correlation with miR-367-5p in GC tissues (Fig. $2 \mathrm{~g}, r=0.641, P<0.0001$ ). These results suggested that circYAP1 might bind with much more miR-367-5p, resulting in the decrease of free miR-367-5p.

\section{CircYAP1 inhibits the proliferation and invasion of GC cells}

To investigate the biological functions of circYAP1 in GC cells, we performed qPCR to measure the expression level of circYAP1 in GC cells. CircYAP1 levels were lower in GC cells than GES-1 cells. AGS cells showed the lowest expression level, MKN-45 cells showed a moderate expression level, and HGC-27 cells showed the highest expression level (Additional file 3: Figure S2a). Therefore, AGS and MKN-45 cells were chosen for circYAP1 overexpression, and HGC-27 cells were selected for circYAP1 silencing. We constructed circYAP1-overexpressing lentiviruses with circular frames and circYAP1 sequences and found that circYAP1 was significantly overexpressed in AGS and MKN-45 cells (Additional file 3: Figure S2b and c). Moreover, miR-367-5p mimics were successfully transfected into the AGS and MKN-45 GC cells (Additional file 3: Figure S2d and e). In addition, the circYAP1 expression level 


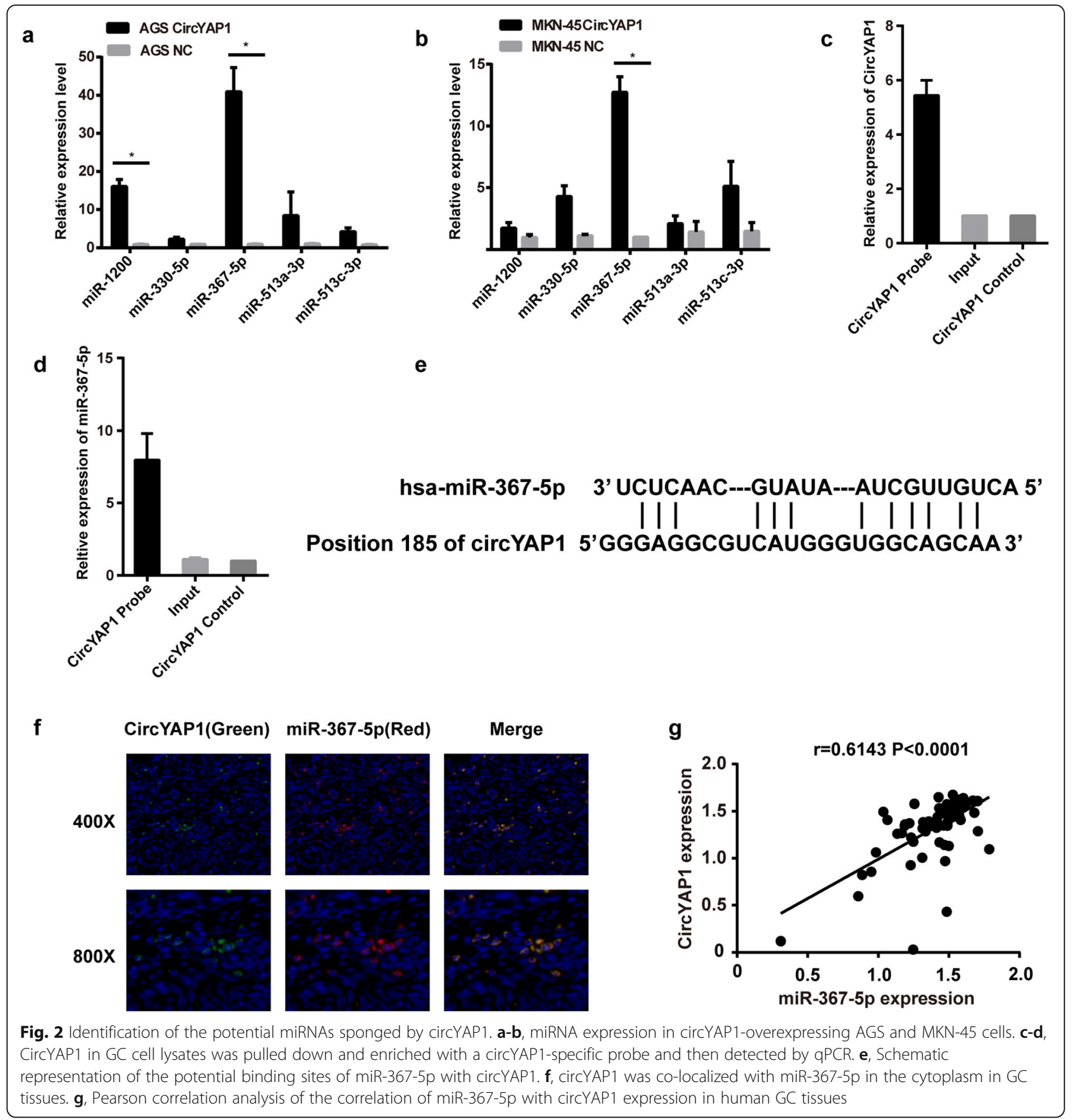

transfected with miR-367-5p mimics was similar to those transfected with mimics NC (Additional file 3: Figure S2f).

The effects of circYAP1 overexpression on cell proliferation, invasion and cycle distribution were determined by 5-ethynyl-2'-deoxyuridine (EdU), clone formation, Transwell assays and flow cytometry analysis. Cell proliferation assay showed that circYAP1 overexpression significantly suppressed AGS and MKN-45 cell growth (Fig. 3a and b). Cell cycle distribution assays also revealed that circYAP1 overexpression induced considerable arrest at the G1 phase in AGS and MKN-45 cells (Additional file 4: Figure S3a and b, $P=$ 0.0038; $P=0.0156)$. However, the effects of cell proliferation suppression and cell cycle arrest induced by circYAP1 were reversed when the cells were co-transfected with miR-367-5p mimics (Fig. 3c-d and Additional file 4: Figure S3a and b). EdU incorporation assays showed that GC cells proliferation was impaired upon circYAP1 overexpression (Fig. 3e and f, $P=0.0172 ; P<0.0001$ ), and this impairment could be rescued by co-transfection 

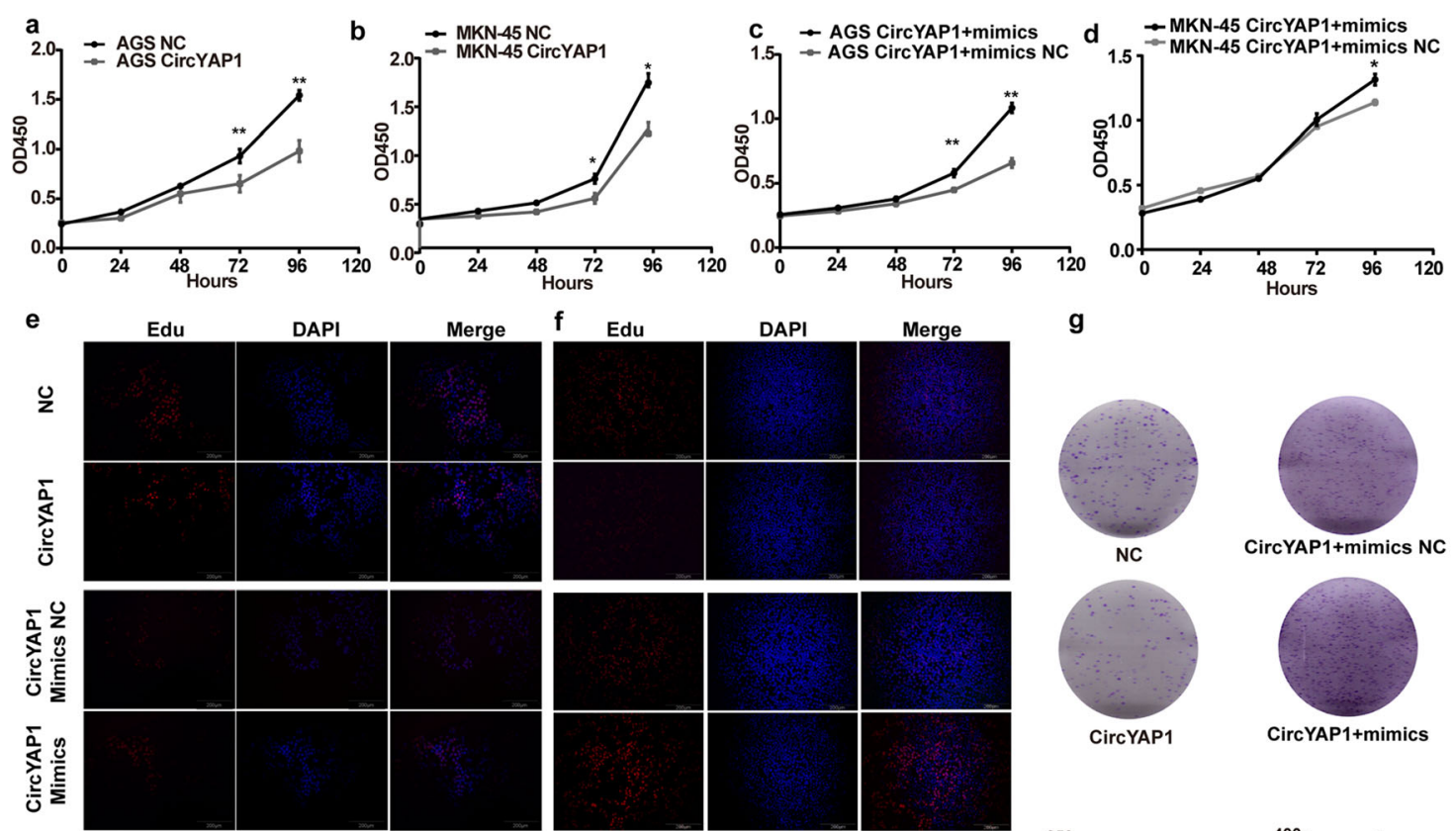

g
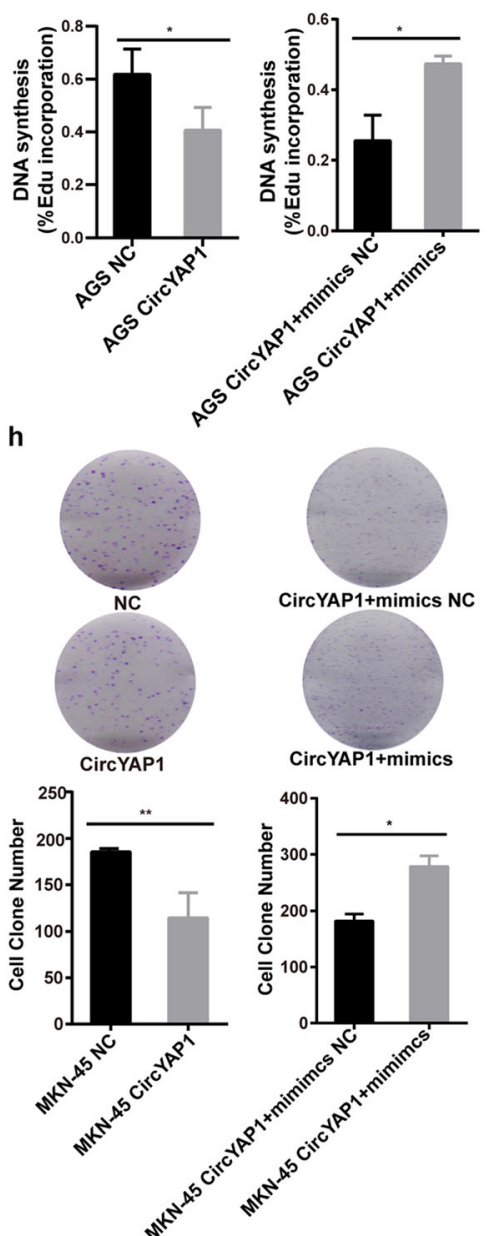
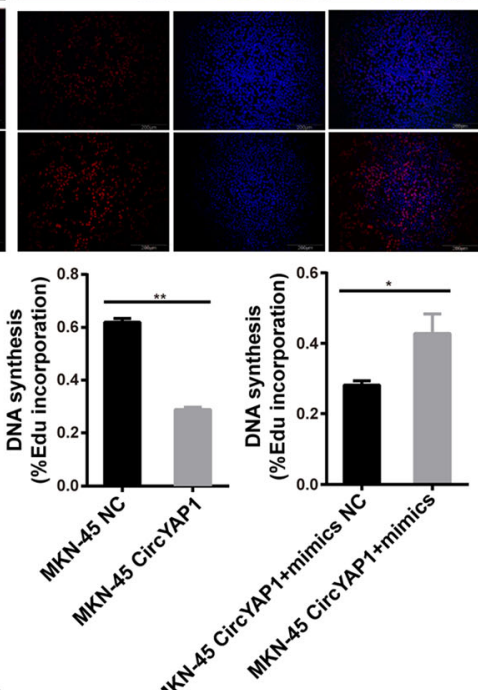

i
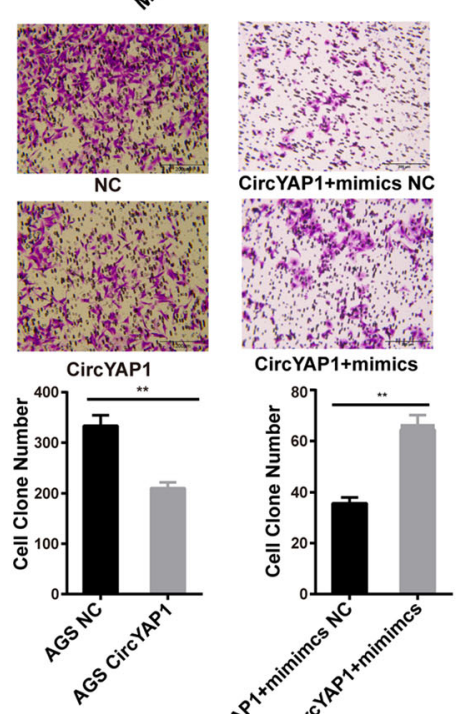

CircYAP1+mimics NC
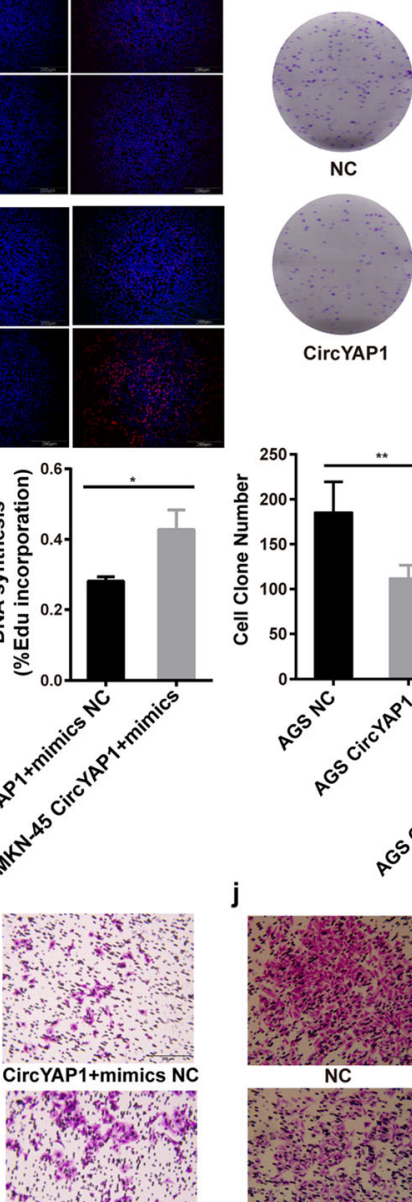

NC

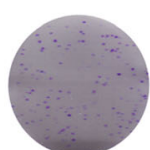

CircYAP1

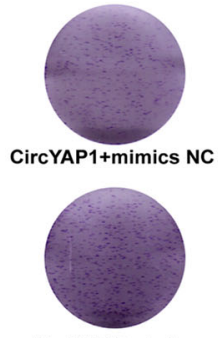

CircYAP1+mimics
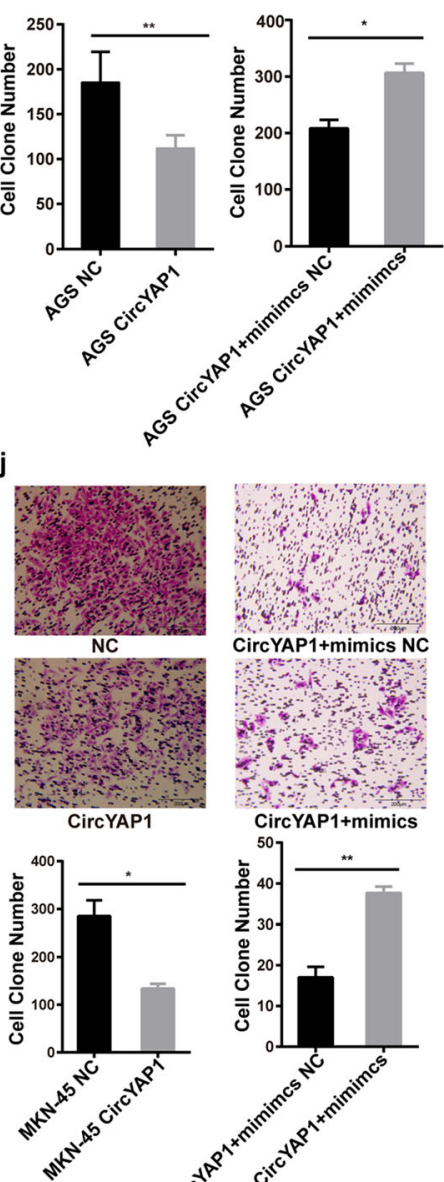

Fig. 3 (See legend on next page.) 
(See figure on previous page.)

Fig. 3 CircYAP1 inhibits proliferation, colony formation and invasion. a-d, Cell proliferation activity and e-f DNA synthesis were assessed in AGS and MKN-45 cells transfected with the circYAP1 overexpression vector or circYAP1 overexpression vector + miR-367-5p mimics. g-h, Clone formation assays were conducted in AGS and MKN-45 cells transfected with the circYAP1 overexpression vector or circYAP1 overexpression vector + miR-367-5p mimics. i-j, Cell invasion potential was determined in AGS and MKN-45 cells transfected with the circYAP1 overexpression vector or circYAP1 overexpression vector + miR-367-5p mimics according to Transwell assays. ${ }^{*} P<0.05 ;{ }^{*} P<0.01$

with the miR-367-5p mimics (Fig. 3e and f, $P=0.0264$; $P=0.0353)$. Furthermore, clone formation assay indicated that the compromised colony-forming ability of these two GC cell lines upon circYAP1 overexpression could return to normal levels when the cells were co-transfected with miR-367-5p mimics (Fig. 3g and h). In addition, the invasion ability of these two types of GC cells was impaired upon circYAP1 overexpression, and this impairment could be rescued by the miR-367-5p mimics (Fig. $3 i$ and j). However, cell apoptosis was not affected by circYAP1 in GC cells (Additional file 2: Figure S1c-f).

\section{CircYAP1 knockdown promotes GC cell proliferation and invasion}

To evaluate the negative biological functions of circYAP1, we used small interference RNA (siRNA) that targeted the back-splice sequence of circYAP1 in HGC-27 cells (Fig. 4a). The siRNA efficiency was confirmed by qPCR (Additional file 3: Figure S2 g). Cell proliferation assays revealed that cell proliferation was enhanced upon circYAP1 silencing (Fig. 4b) and these effects could be ablated by the miR-367-5p inhibitor (Fig. 4c). Cell cycle assays indicated that the proportion of G1 and G2/M cells in the phase decreased upon circYAP1 silencing (Additional file 4: Figure S3c, $P=$ $0.0010 ; P=0.0003$ ). However, the proportion of cells in the $\mathrm{S}$ phase increased when the cells were co-transfected with miR-367-5p inhibitor (Additional file 4: Figure S3c, $P=0.0002 ; P=0.0003$ ). EdU incorporation assays showed that cell proliferation was enhanced upon circYAP1 knockdown (Fig. 4d, $P=0.0064$ ) and these effects could be neutralized by the miR-367-5p inhibitor (Fig. $4 \mathrm{~d}, P=0.0002$ ). Clone formation assays revealed that circYAP1 knockdown promoted the colony-forming ability of HGC-27 cells (Fig. 4e, $P=0.0056$ ), but these effects were weakened when the cells were co-transfected with the miR-367-5p inhibitor (Fig. 4e, $P=0.0004$ ). Furthermore, cell invasion assays showed that the invasion ability of HGC-27 was enhanced by circYAP1 knockdown $(P=0.0009)$, but this effect was reversed by co-transfected with the miR-367-5p inhibitor (Fig. 4f, $P=0.0050)$.

\section{$\mathrm{P} 27^{\mathrm{Kip} 1}$ was validated as a target gene of miR-367-5p}

Next, we used Targetscan (http://www.targetscan.org/ vert_71/) to predict the target genes of miR-367-5p; and the gene gene-p27 Kip1 had the best potential (Fig. 5a). 293T cells co-transfected with plasmid containing $3^{\prime}$ '-UTR-WT regions of p27 Kip1 and miR-367-5p mimics had significantly less relative luciferase activity than the controls, while mutation of the potential miR-367-5p binding sites in the p27 Kip1 $3{ }^{\prime}$-UTR abolished the effect (Fig. 5b, $p=0.0029$ ). It was indicated that $\mathrm{p} 27{ }^{\text {Kip } 1}$ might be a putative target of miR-367-5p.

\section{CircYAP1 sponged miR-367-5p to upregulate $p 27^{\text {Kip1 }}$}

To determine whether circYAP1 sponges miR-367-5p to regulate p27 Kip1 expression, we measured p27 Kip1 ${ }^{\text {ex- }}$ pression level by Western Blotting [19-22]. CircYAP1 overexpression considerably upregulated p27 Kip1 expression in GC cells, and this effect could be reversed by miR-367-5p mimics (Fig. 5c). In addition, silencing circYAP1 decreased the expression of p27 Kip1 and this effect could be also rescued by the miR-367-5p inhibitor (Fig. 5d).

\section{CircYAP1 inhibits tumor growth in vivo}

To further explore whether circYAP1 influences tumor growth in vivo, we constructed circYAP1 overexpression or negative control (NC) stably transfected MKN-45 cells were subcutaneously injected into the flank of nude mice. After 30 days, the tumor volumes were significantly lower in circYAP1-overexpressing MKN-45 cells than in NC-transfected cells (Fig. 6a, b). Statistical analyses showed that the tumor volume was much smaller in the circYAP1-overexpressing group than in the NC-transfected group (Fig. 6c, $P=0.0432$ ). HE staining showed that the tumor formation ability of circYAP1-overexpressing group was lowered as compared with the NC-transfected group (Fig. 6d). IHC analyses showed that the Ki-67 expression was decreased by circYAP1 overexpression (Fig. 6e).

\section{Discussion}

CircRNAs are a new group of ncRNAs with largely unclear biological functions. The roles of circRNAs in carcinogenesis have recently attracted extensive attention. It has been reported that circRNAs are aberrantly expressed in tumors and function as tumor suppressors or oncogenes in the progression of cancer pathogenesis [5, 23, 24]. For example, circCCDC66, circMTO1, circMYLK and circHIAT1 promote tumor growth and metastasis $[13,14,16,25]$, while circLARP4 and circZKSCAN1 suppress tumor progression by multiple 
a

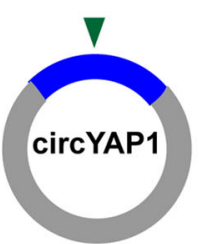

Si-CirCYAP1:CGGCAGGUCCUCUUCCUGATTT

b

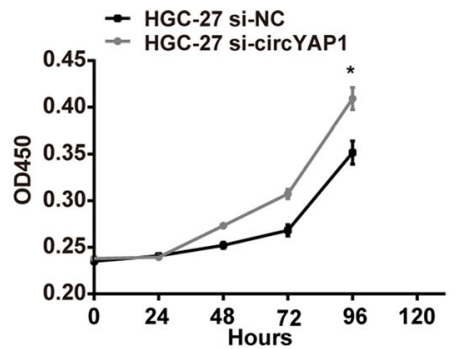

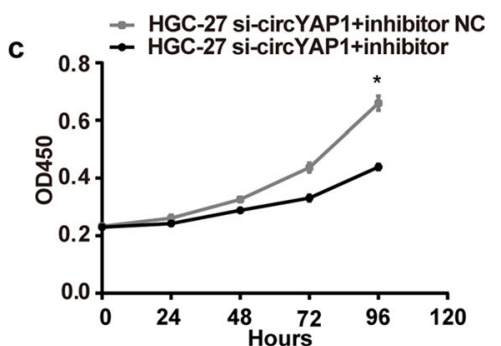

d
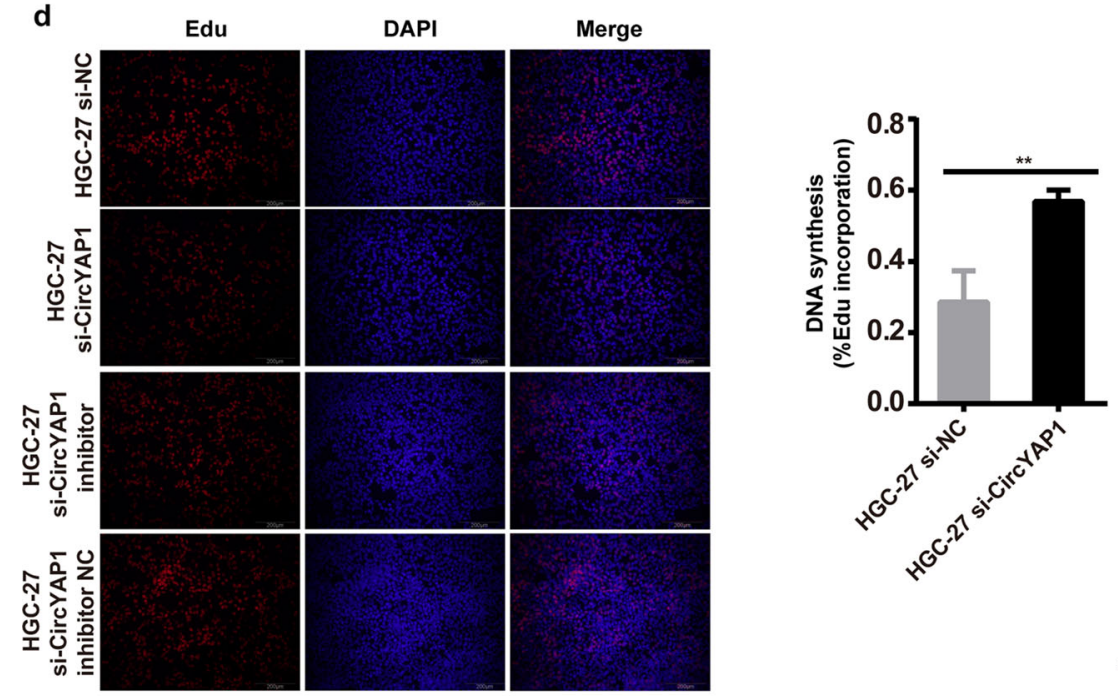

e
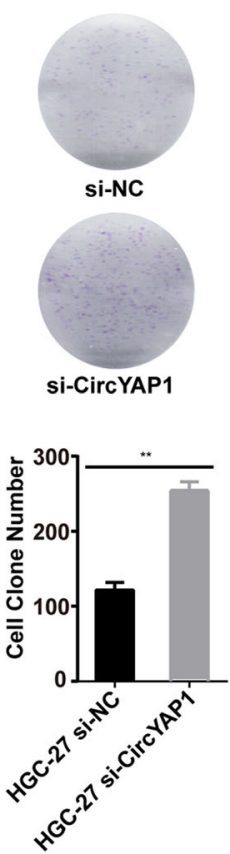

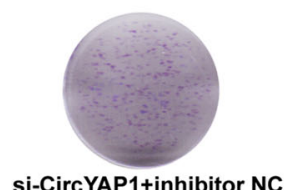

si-CircYAP1+inhibitor

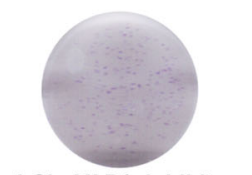

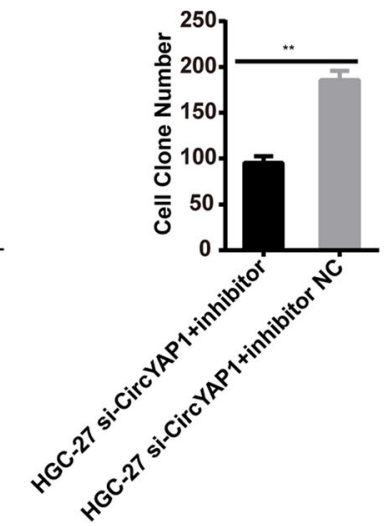
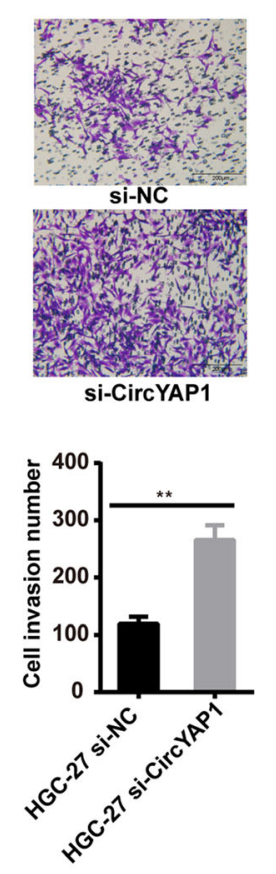

\section{f}

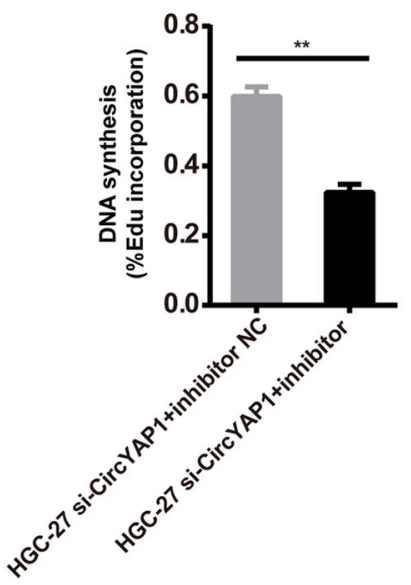

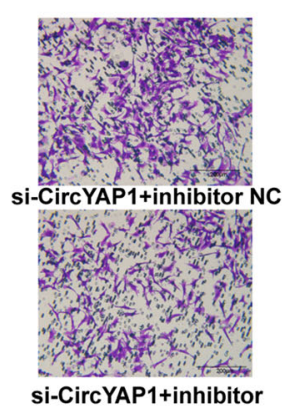

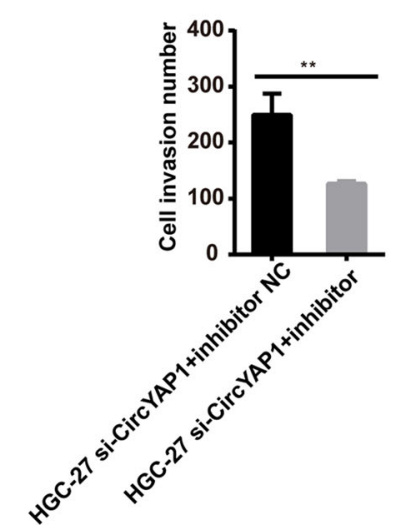

Fig. 4 (See legend on next page.) 
(See figure on previous page.)

Fig. 4 Knockdown circYAP1 promotes proliferation, colony formation and migration. a, Schematic representation of the target sequences of the siRNAs specific to the back-splicing junction of circYAP1. b-c, Cell proliferation activity and d, DNA synthesis in HGC-27 cells transfected with si-circYAP1 or si-circYAP1 + miR-367-5p inhibitor. e, Clone formation assays of HGC-27 cells transfected with si-circYAP1 or si-circYAP1 + miR-367$5 p$ inhibitor. $\mathbf{f}$, Cell invasion potential of HGC-27 cells transfected with si-circYAP1 or si-circYAP1 + miR-367-5p inhibitor according to Transwell assays. ${ }^{*} P<0.05 ;{ }^{*} P<0.01$

complicated signaling pathways $[26,27]$. Here, we combined the reported literature with a CirNet analysis to find circYAP1, which is derived from the YAP1 gene locus [28]; Many studies have revealed that YAP1 enhances cell proliferation, migration and invasion in GC $[29,30]$. However, the expression and functional role of circYAP1 in GC remain unknown. We investigated 17 GC tissues and paired adjacent normal tissues and found that circYAP1 expression was significantly higher in adjacent normal tissues than in matched GC tissues. It was suggested that circYAP1 might act as an anti-oncogene rather than an oncogene in GC. This result was further verified in another 80 cases of GC tissues.

Decreased circYAP1 expression in GC was significantly correlated with poor prognosis in GC patients. GC patients with high circYAP1 expression had longer survival times than those with low circYAP1 expression. CircRNA expression was dynamically changed with the development of the disease. CircYAP1 expression levels were higher in the early-stage GC patients than in the late-stage GC patients. In addition, GC patients with high circYAP1 expression levels were more sensitive to chemotherapy than those with low circYAP1 expression levels. It was illustrated that circYAP1 could act as a biomarker for predicting the survival or an index for chemotherapy responses in GC patients.

CircRNAs in different types of cancer or different stages vary in regulating the gene expression [31]. CircRNAs are well known to serve as miRNA sponges to form the circRNA-miRNA-mRNA axis $[8,32]$. For example, our previous study revealed that circLARP4 sponges miR-424-5P to activate LATS1-YAP signaling in GC [27]. CircCCDC66 sponges multiple miRNAs to promote $\mathrm{CRC}$ growth and metastasis [14]. CircMOT1 sponges miR-9 to suppress HCC progression [13], circMYLK sponges miR-29a to activate the VEGFA/VEGFR2 pathway to promote bladder cancer progression [16], and ciRS-7 is involved in a variety of cancers by its interaction with miR-7 [33].

In this study, CircNet and CircInteractome were used to predict circRNAs [34], and the circRNAs were selected with the relatively unbiased approach of computational algorithms. As reported. ciRS-7 is resistant to the conventional miRNA destabilization of mRNA and therefore not prone to miR-7-dependent regulation. miR-7 and ciRS-7 display co-expression in cells. The high expression of ciRS-7 coincides with miR-7 expression in mouse brain

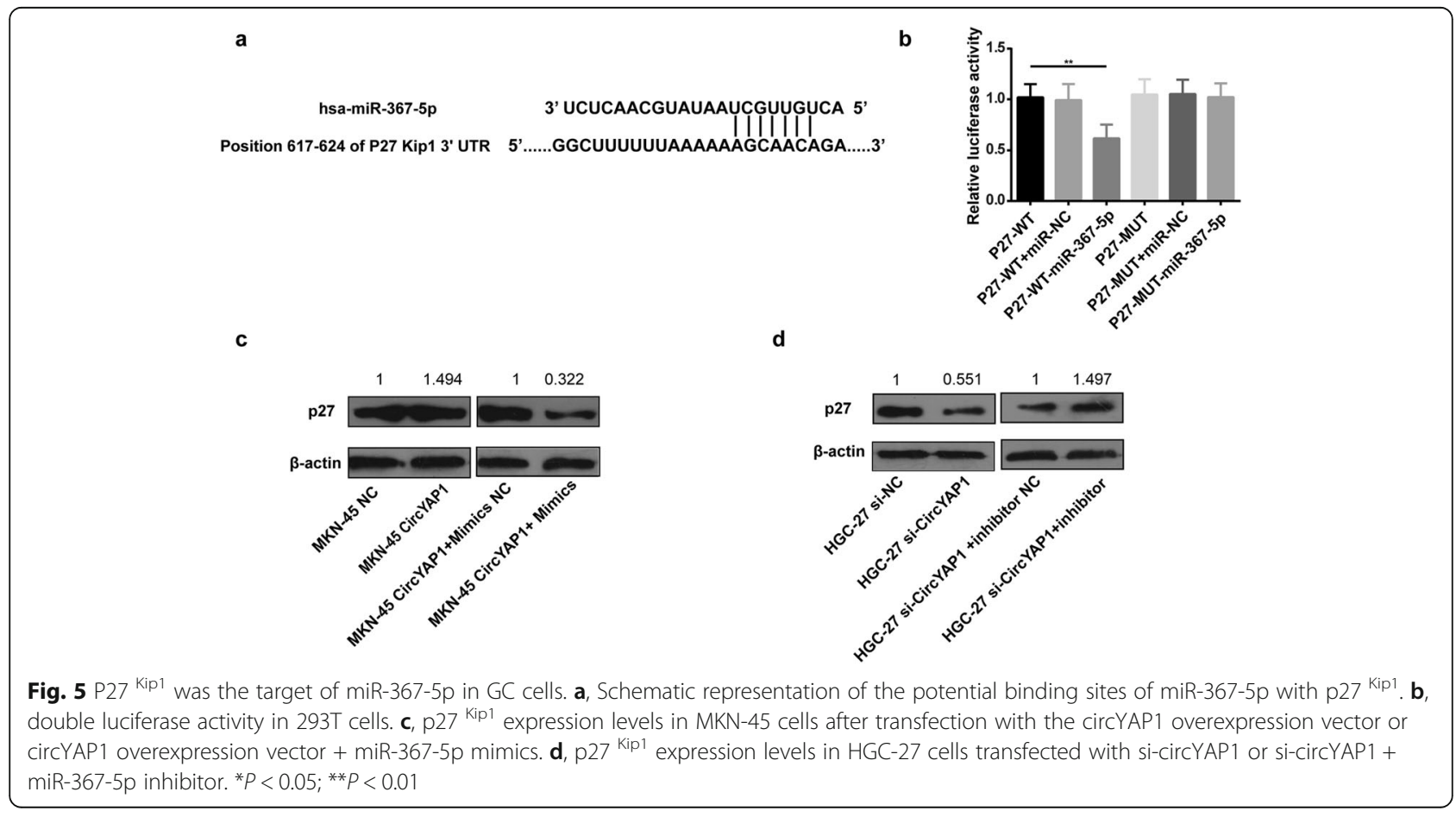



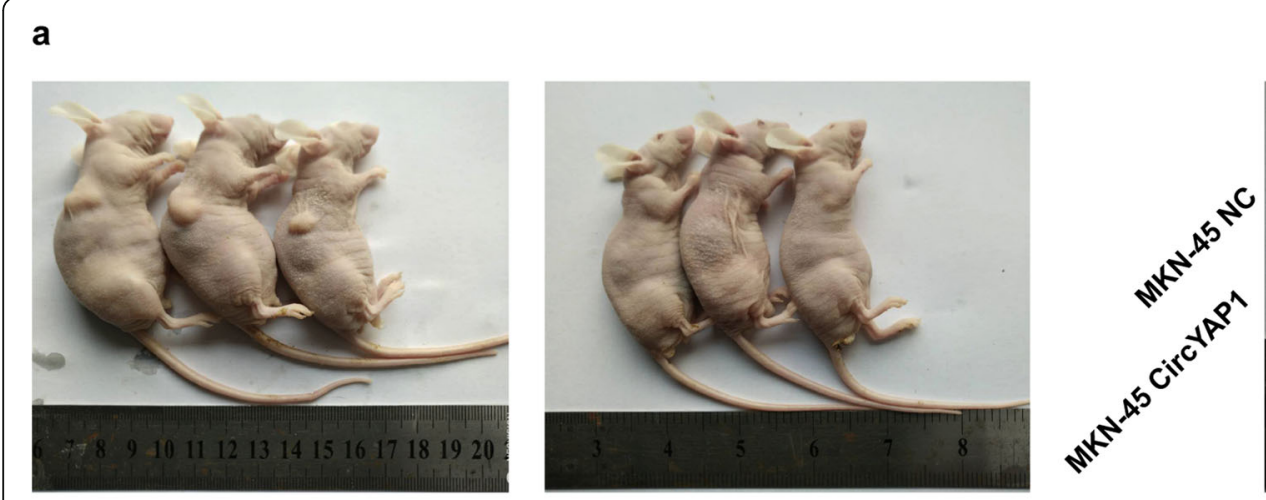

b

MKN-45 NC

MKN-45 CircYAP1

C

d

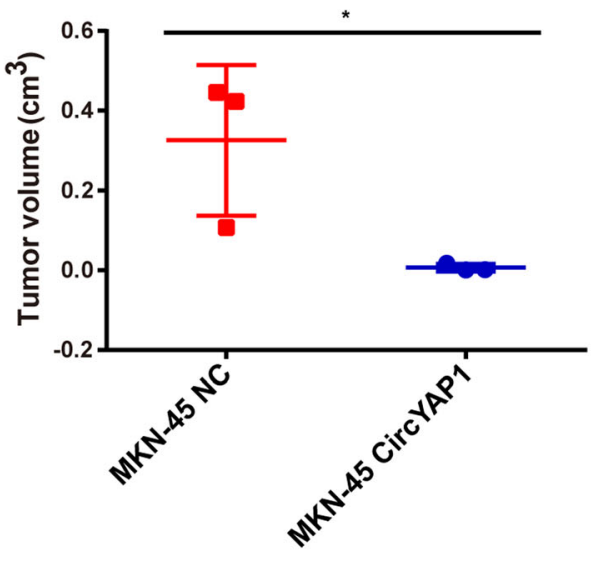

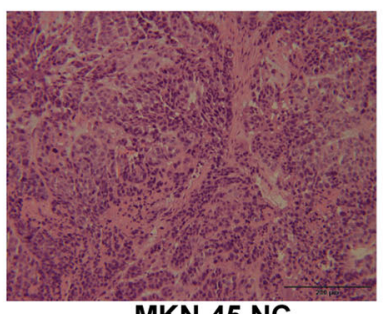

MKN-45 NC

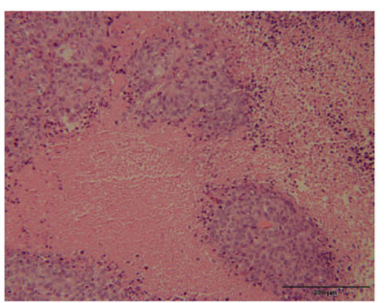

MKN-45 CircYAP1

e

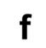

11q22.1(101981192....102104154)

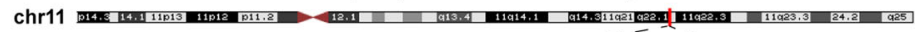
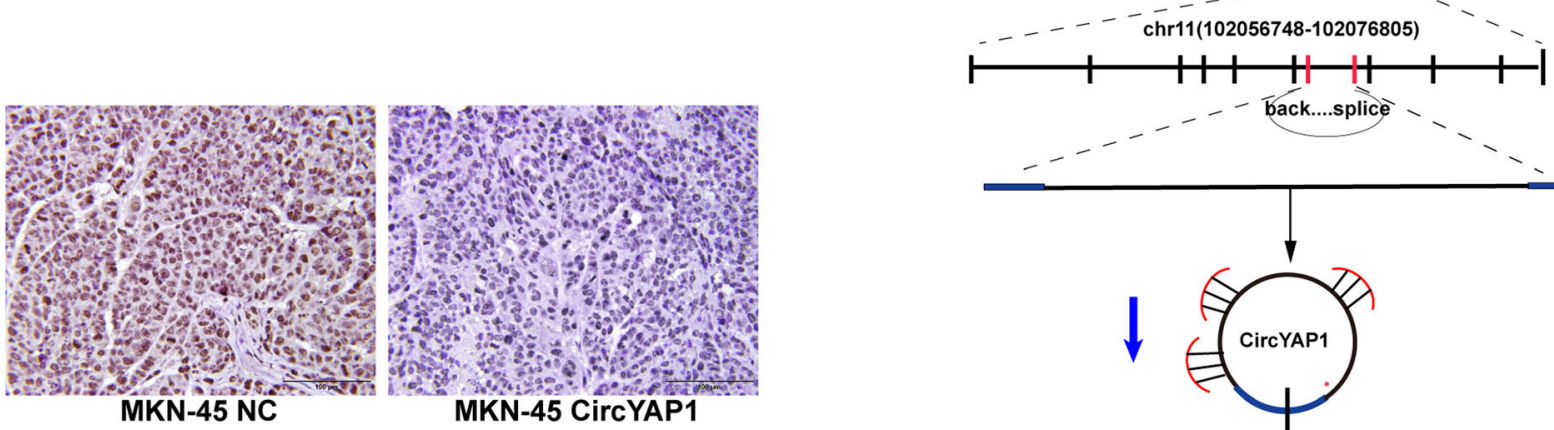

F
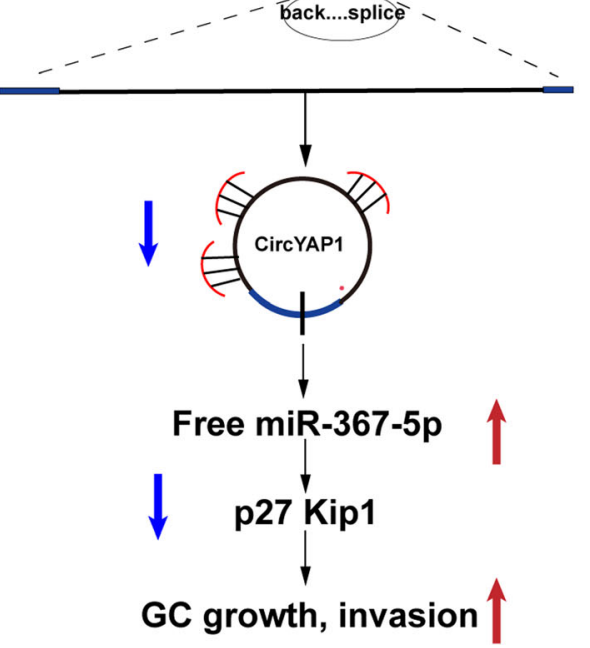

Fig. 6 (See legend on next page.) 
(See figure on previous page.)

Fig. 6 CircYAP1 inhibited tumor growth in vivo. a, Nude mice were subcutaneously injected with circYAP1-overexpressing MKN-45 cells and NC cells. b. Schematic representation of the MKN-45 xenograft tumor size after 30 days of tumor growth in the circYAP1 overexpression and NC groups. c, Statistical comparison of the differences in tumor volumes between the circYAP1 overexpression and NC groups. $\mathbf{d}$, HE analysis of the tumor formation in circYAP1 overexpression and NC groups. e, $\mathrm{HC}$ analysis of Ki-67 expression levels in xenograft tumor tissues from the circYAP1 overexpression and NC groups $(\times 400)$. f, Loss of circYAP1 sponges and increases the free miR-367-5p levels, and thereby decreased p27 Kip1 expression, leading to the GC tumorigenesis

sections and in primary cells isolated from mouse brain, which strongly suggests that miR-7 is interacting endogenously with ciRS-7. CDR1as has been proposed to function as a sponge for miR-7 by reducing the number of freely available miR-7 molecules [8]. Other studies also revealed that the loss of circRNAs causes miRNA degeneration [35]. Thus, we measured the expression levels of the miRNAs with the potential to bind with circYAP1 and identified that miR-367-5p was significantly upregulated by circYAP1 in GC cells. Then, we performed circRIP using a circYAP1-specific probe and found that miR-367-5p was the circYAP1-binding miRNA. We proved that circYAP1 sponged miR-367-5p in GC cells. Further functional experiments showed that circYAP1 inhibited GC cell growth, invasion and cycle progression in vitro and in vivo, but knockdown of circYAP1 had the opposite effects, suggesting that circYAP1 might act as a tumor suppressor in GC.

MiRNA, a key component of the ncRNA family, plays multifaceted roles in controlling cellular functions by repressing target genes [36]. miR-367 has been reported as an oncogene in uveal melanoma cells [37], osteosarcoma [38], medulloblastoma [39] and non-small cell lung cancer [40, 41]. In our study, we also found that miR-367-5p acted as an oncogene in GC cells. p27 Kip1 is a cyclin-dependent kinase (CDK) inhibitor that regulates cell proliferation, cell motility and apoptosis. Low expression of $\mathrm{p} 27^{\mathrm{Kip} 1}$ is correlated with high-grade tumors and poor prognosis in several types of human cancer [42]. More importantly, studies also described an association between $\mathrm{p} 27^{\mathrm{Kip} 1}$ loss and poor prognosis in gastric cancer [20, 43]. CircYAP1 upregulated the expression level of p27 Kip1, and this effect was abolished by co-transfection with miR-367-5p. CircYAP1 knockdown suppressed p27 Kip1 expression, and the effects could be reversed when the cells were co-transfected with a miR-367-5p inhibitor. The luciferase activity assay also exhibited the binding of miR-367-5p with $\mathrm{p} 27^{\mathrm{Kip} 1}$. It was demonstrated that $\mathrm{p} 27^{\mathrm{Kip} 1}$ is the target of miR-367-5p and circYAP1 sponges miR-367-5p to upregulates p27 Kip1 expression in GC cells.

\section{Conclusions}

In summary, our findings reveal that circYAP1 expression is significantly decreased in GC and low circYAP1 expression is associated with a poor prognosis in GC patients. Functionally, circYAP1 inhibits GC cell growth and invasion by sponging miR-367-5p to upregulate p27 Kip1. We speculate the loss of circYAP1 sponges less miR-367-5p and increases the free miR-367-5p in GC cells, and thereby decreases p27 ${ }^{\mathrm{Kip} 1}$ expression, contributing to the GC tumorigenesis (Fig. 6f). CircYAP1 may have considerable potential as a prognostic predictor and therapeutic target for GC.

\section{Additional files}

Additional file 1: Tables S1. Sequences of primers in the study. Table S2. Clinic-pathological data of GC patients from Tissue Microarray. Table S3. Correlation of circYAP1 expression with clinic-pathologic characteristics of GC patients. Table S4. Summary of univariate and multivariate Cox regression analysis of overall survival duration (DOCX 20 kb)

Additional file 2: Figure S1. a, The expression levels of circYAP1 in GC patients with $T S \geq 5 \mathrm{~cm}$ or $<5 \mathrm{~cm}$. $\mathbf{b}$, Receiver operating characteristic (ROC) curve analysis of the cutoff value, sensitivity, specificity and AUC of circYAP1 in GC patients. c-f, Flow cytometry was used to detect the proportion of apoptotic cells in circYAP1-overexpression-transfected MKN-45 cells (PDF $241 \mathrm{~kb}$ )

Additional file 3: Figure S2. a, CircYAP1 expression in GES-1 and GC cell lines. b-c, AGS and MKN-45 GC cells transfected with the circYAP1 overexpression lentivirus. d-e, miR-367-5p mimics were transfected into AGS and MKN-45 GC cells. f, qPCR analysis of the transfection efficiency of si-circYAP1 vectors after transfection for $48 \mathrm{~h}$ in $\mathrm{HGC}-27$ cells. ${ }^{*} P<0.05$; ${ }^{* *} P<0.01$ (PDF $619 \mathrm{~kb}$ )

Additional file 4: Figure S3. Cell cycle analysis. a, Cell cycle assays of AGS transfected with circYAP1 or circYAP1 + miR-367-5p mimics. b Cell cycle assays of MKN-45 transfected with circYAP1 or circYAP1 + miR-367$5 p$ mimics. c Cell cycle assays of HGC-27 cells transfected with si-circYAP1 or si-circYAP1 + miR-367-5p inhibitor. ${ }^{*} P<0.05$; ${ }^{* *} P<0.01$ (PDF $1324 \mathrm{~kb}$ )

\section{Abbreviations}

circRIP: CircRNA in vivo precipitation; circRNA: Circular RNA; EdU: 5-Ethynyl20-deoxyuridine; GC: gastric cancer; miRNA: MicroRNA; qRT-PCR: Quantitative real-time polymerase chain reaction; YAP1: Yes-associated protein 1

\section{Acknowledgements}

Not applicable

\section{Funding}

This study was supported by grants from the National Natural Science Foundation of China (No. 81573747 and No. 81470833).

\section{Availability of data and materials}

All data generated or analysed during this study are included in this published article [and its Additional files].

\section{Authors' contributions}

JSZ and JZ contributed to the study design and literature guide. HL contributed to carrying out experiments and writing. ZLB contributed to study supervision and revising the manuscript critically for important 
intellectual content during the manuscript revising progress. $\mathrm{HL}$ and $\mathrm{ZLB}$ contributed to Figures creation. YL contributed to the statistical analysis. The authors are also grateful to RZ who helped conduct manuscript preparation, and YW, YXH, XYC who helped conduct the data collection. All authors read and approved the final manuscript.

\section{Ethics approval and consent to participate}

The research was approved by the Ethics Committee of Shanghai Jiao Tong University Affiliated Sixth People's Hospital and Nantong Third People's Hospital Affiliated to Nantong University.

\section{Consent for publication}

Not applicable

\section{Competing interests}

The authors declare that they have no competing interests.

\section{Publisher's Note}

Springer Nature remains neutral with regard to jurisdictional claims in published maps and institutional affiliations.

\section{Author details}

'Department of Gastroenterology, Shanghai Jiao Tong University Affiliated Sixth People's Hospital, No. 600 Yishan Road, Shanghai 200233, China. ${ }^{2}$ Nantong Institute of Liver Disease, Department of Gastroenterology and Hepatology, Nantong Third People's Hospital, Nantong University, Nantong 226006, Jiangsu, China. ${ }^{3}$ Medical School of Nantong University, Nantong 226006, Jiangsu, China.

Received: 12 May 2018 Accepted: 8 October 2018 Published online: 18 October 2018

\section{References}

1. Torre LA, Bray F, Siegel RL, Ferlay J, Lortet-Tieulent J, Jemal A. Global cancer statistics, 2012. CA Cancer J Clin. 2015;65:87-108.

2. Allemani C, Weir HK, Carreira H, Harewood R, Spika D, Wang XS, Bannon F, Ahn JV, Johnson CJ, Bonaventure A, et al. Global surveillance of cancer survival 1995-2009: analysis of individual data for 25,676,887 patients from 279 population-based registries in 67 countries (CONCORD-2). Lancet. 2015; 385:977-1010.

3. Wang J, Song Y-X, Wang Z-N. Non-coding RNAs in gastric cancer. Gene 2015;560:1-8.

4. Song JH, Meltzer SJ. MicroRNAs in pathogenesis, diagnosis, and treatment of gastroesophageal cancers. Gastroenterology. 2012;143:35-47 e32.

5. Meng S, Zhou H, Feng Z, Xu Z, Tang Y, Li P, Wu M. CircRNA: functions and properties of a novel potential biomarker for cancer. Mol Cancer. 2017:16:94.

6. Salzman J, Chen RE, Olsen MN, Wang PL, Brown PO. Cell-type specific features of circular RNA expression. PLoS Genet. 2013;9:e1003777.

7. Jeck WR, Sorrentino JA, Wang K, Slevin MK, Burd CE, Liu J, Marzluff WF, Sharpless NE. Circular RNAs are abundant, conserved, and associated with ALU repeats. RNA. 2013:19:141-57.

8. Memczak S, Jens M, Elefsinioti A, Torti F, Krueger J, Rybak A, Maier L, Mackowiak SD, Gregersen LH, Munschauer M, et al. Circular RNAs are a large class of animal RNAs with regulatory potency. Nature. 2013;495:333-8.

9. Cortes-Lopez M, Miura P. Emerging functions of circular RNAs. Yale J Bio Med. 2016;89:527-37.

10. Barrett SP, Salzman J. Circular RNAs: analysis, expression and potential functions. Development. 2016;143:1838-47.

11. Li Z, Huang C, Bao C, Chen L, Lin M, Wang X, Zhong G, Yu B, Hu W, Dai L, et al. Exon-intron circular RNAs regulate transcription in the nucleus. Nat Struct Mol Biol. 2015;22:256-64.

12. Chen Y, Li C, Tan C, Liu X. Circular RNAs: a new frontier in the study of human diseases. J Med Genet. 2016;53:359-65

13. Han D, Li J, Wang H, Su X, Hou J, Gu Y, Qian C, Lin Y, Liu X, Huang M, et al. Circular RNA circMTO1 acts as the sponge of microRNA-9 to suppress hepatocellular carcinoma progression. Hepatology. 2017:66:1151-64.

14. Hsiao KY, Lin YC, Gupta SK, Chang N, Yen L, Sun HS, Tsai SJ. Non-coding effects of circular RNA CCDC66 promote colon cancer growth and metastasis. Cancer Res. 2017;77:2339-50.
15. Chen L, Zhang S, Wu J, Cui J, Zhong L, Zeng L, Ge S. circRNA_100290 plays a role in oral cancer by functioning as a sponge of the miR-29 family. Oncogene. 2017;36:4551-61.

16. Zhong Z, Huang M, Lv M, He Y, Duan C, Zhang L, Chen J. Circular RNA MYLK as a competing endogenous RNA promotes bladder cancer progression through modulating VEGFANEGFR2 signaling pathway. Cancer Lett. 2017:403:305-17.

17. Li F, Zhang L, Li W, Deng J, Zheng J, An M, Lu J, Zhou Y. Circular RNA ITCH has inhibitory effect on ESCC by suppressing the Wnt/beta-catenin pathway. Oncotarget. 2015;6:6001-13.

18. Su X, Wang H, Ge W, Yang M, Hou J, Chen T, Li N, Cao X. An in vivo method to identify microRNA targets not predicted by computation algorithms: p21 targeting by miR-92a in Cancer. Cancer Res. 2015;75:2875-85.

19. Kim YK, Yu J, Han TS, Park SY, Namkoong B, Kim DH, Hur K, Yoo MW, Lee HJ, Yang HK, Kim VN. Functional links between clustered microRNAs: suppression of cell-cycle inhibitors by microRNA clusters in gastric cancer. Nucleic Acids Res. 2009;37:1672-81.

20. Sun M, Liu XH, Li JH, Yang JS, Zhang EB, Yin DD, Liu ZL, Zhou J, Ding Y, Li SQ, et al. MiR-196a is upregulated in gastric cancer and promotes cell proliferation by downregulating p27(kip1). Mol Cancer Ther. 2012;11:842-52.

21. Yan K, Tian J, Shi W, Xia H, Zhu Y. LncRNA SNHG6 is associated with poor prognosis of gastric Cancer and promotes cell proliferation and EMT through epigenetically silencing p27 and sponging miR-101-3p. Cell Physiol Biochem. 2017:42:999-1012.

22. Zhang Q, Hou D, Luo Z, Chen P, Lv B, Wu L, Ma Y, Chu Y, Liu H, Liu F, et al. The novel protective role of P27 in MLN4924-treated gastric cancer cells. Cell Death Dis. 2015;6:e1867.

23. He J, Xie Q, Xu H, Li J, Li Y. Circular RNAs and cancer. Cancer Lett. 2017;396: 138-44.

24. Kristensen LS, Hansen TB, Veno MT, Kjems J. Circular RNAs in cancer: opportunities and challenges in the field. Oncogene. 2017;37:555-65.

25. Wang $K$, Sun $Y$, Tao W, Fei $X$, Chang $C$. Androgen receptor (AR) promotes clear cell renal cell carcinoma ( $c C R C C)$ migration and invasion via altering the circHIAT1/miR-195-5p/29a-3p/29c-3p/CDC42 signals. Cancer Lett. 2017:394:1-12.

26. Yao Z, Luo J, Hu K, Lin J, Huang H, Wang Q, Zhang P, Xiong Z, He C, Huang $Z$, et al. ZKSCAN1 gene and its related circular RNA (circZKSCAN1) both inhibit hepatocellular carcinoma cell growth, migration, and invasion but through different signaling pathways. Mol Oncol. 2017;11:422-37.

27. Zhang J, Liu H, Hou L, Wang G, Zhang R, Huang Y, Chen X, Zhu J. Circular RNA_ LARP4 inhibits cell proliferation and invasion of gastric cancer by sponging miR424-5p and regulating LATS1 expression. Mol Cancer. 2017;16:151.

28. Rybak-Wolf A, Stottmeister C, Glazar P, Jens M, Pino N, Giusti S, Hanan M, Behm M, Bartok O, Ashwal-Fluss R, et al. Circular RNAs in the mammalian brain are highly abundant, conserved, and dynamically expressed. Mol Cell. 2015:58:870-85.

29. Kang W, Tong JH, Chan AW, Lee TL, Lung RW, Leung PP, So KK, Wu K, Fan $D, Y u J$, et al. Yes-associated protein 1 exhibits oncogenic property in gastric cancer and its nuclear accumulation associates with poor prognosis. Clin Cancer Res. 2011;17:2130-9.

30. Kang W, Tong JH, Lung RW, Dong Y, Zhao J, Liang Q, Zhang L, Pan Y, Yang W, Pang JC, et al. Targeting of YAP1 by microRNA-15a and microRNA-16-1 exerts tumor suppressor function in gastric adenocarcinoma. Mol Cancer. 2015;14:52.

31. Du WW, Yang W, Liu E, Yang Z, Dhaliwal P, Yang BB. Foxo3 circular RNA retards cell cycle progression via forming ternary complexes with p21 and CDK2. Nucleic Acids Res. 2016:44:2846-58.

32. Hansen TB, Jensen TI, Clausen BH, Bramsen JB, Finsen B, Damgaard CK, Kjems J. Natural RNA circles function as efficient microRNA sponges. Nature. 2013:495:384-8.

33. Hansen TB, Kjems J, Damgaard CK. Circular RNA and miR-7 in cancer. Cancer Res. 2013;73:5609-12.

34. Dudekula DB, Panda AC, Grammatikakis I, De S, Abdelmohsen K, Gorospe M Circlnteractome: a web tool for exploring circular RNAs and their interacting proteins and microRNAs. RNA Biol. 2016:13:34-42.

35. Piwecka M, Glazar P, Hernandez-Miranda LR, Memczak S, Wolf SA, RybakWolf A, Filipchyk A, Klironomos F, Cerda Jara CA, Fenske P, et al. Loss of a mammalian circular RNA locus causes miRNA deregulation and affects brain function. Science 2017. 357.

36. Rupaimoole R, Calin GA, Lopez-Berestein G, Sood AK. miRNA deregulation in Cancer cells and the tumor microenvironment. Cancer Discov. 2016:6:235-46. 
37. Ling JW, Lu PR, Zhang YB, Jiang S, Zhang ZC. miR-367 promotes uveal melanoma cell proliferation and migration by regulating PTEN. Genet Mol Res. 2017;16.

38. Cai W, Jiang H, Yu Y, Xu Y, Zuo W, Wang S, Su Z. miR-367 regulation of DOC-2/DAB2 interactive protein promotes proliferation, migration and invasion of osteosarcoma cells. Biomed Pharmacother. 2017;95:120-8.

39. Kaid C, Silva PB, Cortez BA, Rodini CO, Semedo-Kuriki P, Okamoto OK. miR367 promotes proliferation and stem-like traits in medulloblastoma cells. Cancer Sci. 2015;106:1188-95.

40. Campayo M, Navarro A, Vinolas N, Diaz T, Tejero R, Gimferrer JM, Molins L, Cabanas ML, Ramirez J, Monzo M, Marrades R. Low miR-145 and high miR367 are associated with unfavourable prognosis in resected nonsmall cell lung cancer. Eur Respir J. 2013;41:1172-8.

41. Xiao G, Zhang B, Meng J, Wang J, XU C, Tang SC, Li X, Zhang J, Liang R, Ren H, Sun X. miR-367 stimulates Wnt cascade activation through degrading FBXW7 in NSCLC stem cells. Cell Cycle. 2017;16:2374-85.

42. Chu IM, Hengst L, Slingerland JM. The Cdk inhibitor p27 in human cancer: prognostic potential and relevance to anticancer therapy. Nat Rev Cancer. 2008:8:253-67.

43. Mori M, Mimori K, Shiraishi T, Tanaka S, Ueo H, Sugimachi K, Akiyoshi T. p27 expression and gastric carcinoma. Nat Med. 1997;3:593.

Ready to submit your research? Choose BMC and benefit from:

- fast, convenient online submission

- thorough peer review by experienced researchers in your field

- rapid publication on acceptance

- support for research data, including large and complex data types

- gold Open Access which fosters wider collaboration and increased citations

- maximum visibility for your research: over $100 \mathrm{M}$ website views per year

At BMC, research is always in progress.

Learn more biomedcentral.com/submissions 